\title{
False Positive Class II HLA Antibody Reaction Due to Antibodies Against Denatured HLA Might Differ Between Assays: One Lambda vs. Immucor
}

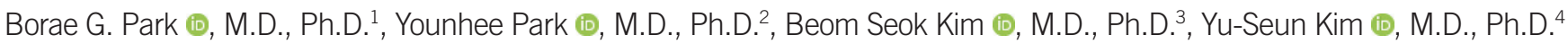 \\ and Hyon-Suk Kim (i), M.D., Ph.D. ${ }^{2}$ \\ ${ }^{1}$ Department of Laboratory Medicine, Korea University Guro Hospital, Seoul, Korea; Departments of ${ }^{2}$ Laboratory Medicine, ${ }^{3}$ Nephrology, and ${ }^{4}$ Transplant \\ Surgery, Severance Hospital, Yonsei University College of Medicine, Seoul, Korea
}

\section{Dear Editor,}

The Luminex human leukocyte antigen (HLA) single-antigen bead assay (SABA) was developed as a sensitive and specific assay to detect HLA antibodies [1]. However, false positive reactions may occur in the SABA due to the effect of medications such as high-dose intravenous immunoglobulin, anti-thymocyte globulin, and anti-CD20 or the presence of antibodies against denatured HLA [1]. Several approaches to overcome this issue have been proposed, including the use of a pretreatment reagent that adsorbs non-specific antibodies or drugs and acid treatment to distinguish specific antibodies against denatured class I HLA [2]. The use of acid treatment and iBead (singleantigen flow bead) has largely resolved the problem caused by antibodies against denatured class I HLA [2, 3]. However, there is no method for distinguishing antibodies against denatured class II HLA. Antibodies against denatured class II HLA have been detected at a frequency of $11 \%$ in healthy male donors [4]. Pan-HLA-DR reactivity is a pattern typically observed in the presence of antibodies against denatured class II HLA [5]. Another study found allelic bead reactions with $H L A-D R B 1{ }^{*} 09: 01$, $D R B 3 * 01: 01, D R B 3 * 02: 02, D R B 3{ }^{*} 03: 01, D P B 1 * 02: 01$, $D P B 1 * 20: 01$, and $D P B 1 * 28: 01$ for antibody reaction against denatured class II HLA [6].

Here, we report a case of false positive reaction with an antibody against denatured class II HLA, which showed different reactivities in screening and while using identification beads and SABA kits from different manufacturers. This study was approved by the Institutional Review Board of Severance Hospital, Seoul, Korea (4-2019-0984).

A 52-year-old female with end-stage renal disease caused by membranous nephropathy visited Severance hospital for her first kidney transplant in April 2016. She had no history of transfusion or desensitization, such as high-dose intravenous immunoglobulin, anti-thymocyte globulin, or anti-CD20, and her pregnancy history was unknown. The patient did not provide informed consent specific to this study but did provide consent for the test and signed a comprehensive agreement on the potential use of the donated sample for research purposes.

Genomic DNA was extracted from the peripheral blood using the QuickGene-Mini80 DNA Isolation System (Fujifilm, Tokyo, Japan). HLA typing was performed without delay using Lifecodes HLA SSO Typing Kits (Immucor Transplant Technology, Stamford, CT, USA), which revealed $H L A-A{ }^{*} 11,{ }^{*} 33 ; B * 27$, *54; DRB1*08, *14, DQB1*05, *08. The result of the initial
Received: October 6, 2019

Revision received: January 3, 2020

Accepted: March 13, 2020

Corresponding author: Younhee Park, M.D., Ph.D.

Department of Laboratory Medicine, Severance Hospital, Yonsei University College of Medicine, 50-1 Yonsei-ro, Seodaemun-gu, Seoul 03722, Korea Tel: +82-2-2228-2440, Fax: +82-2-364-1583, E-mail: younheep@gmail.com

\section{(c) (i) (3)}

\section{(C) Korean Society for Laboratory Medicine}

This is an Open Access article distributed under the terms of the Creative Commons Attribution Non-Commercial License (https://creativecommons.org/licenses/by-nc/4.0) which permits unrestricted non-commercial use, distribution, and reproduction in any medium, provided the original work is properly cited. 
Park BG, et al.

False reaction with denatured HLA antibody
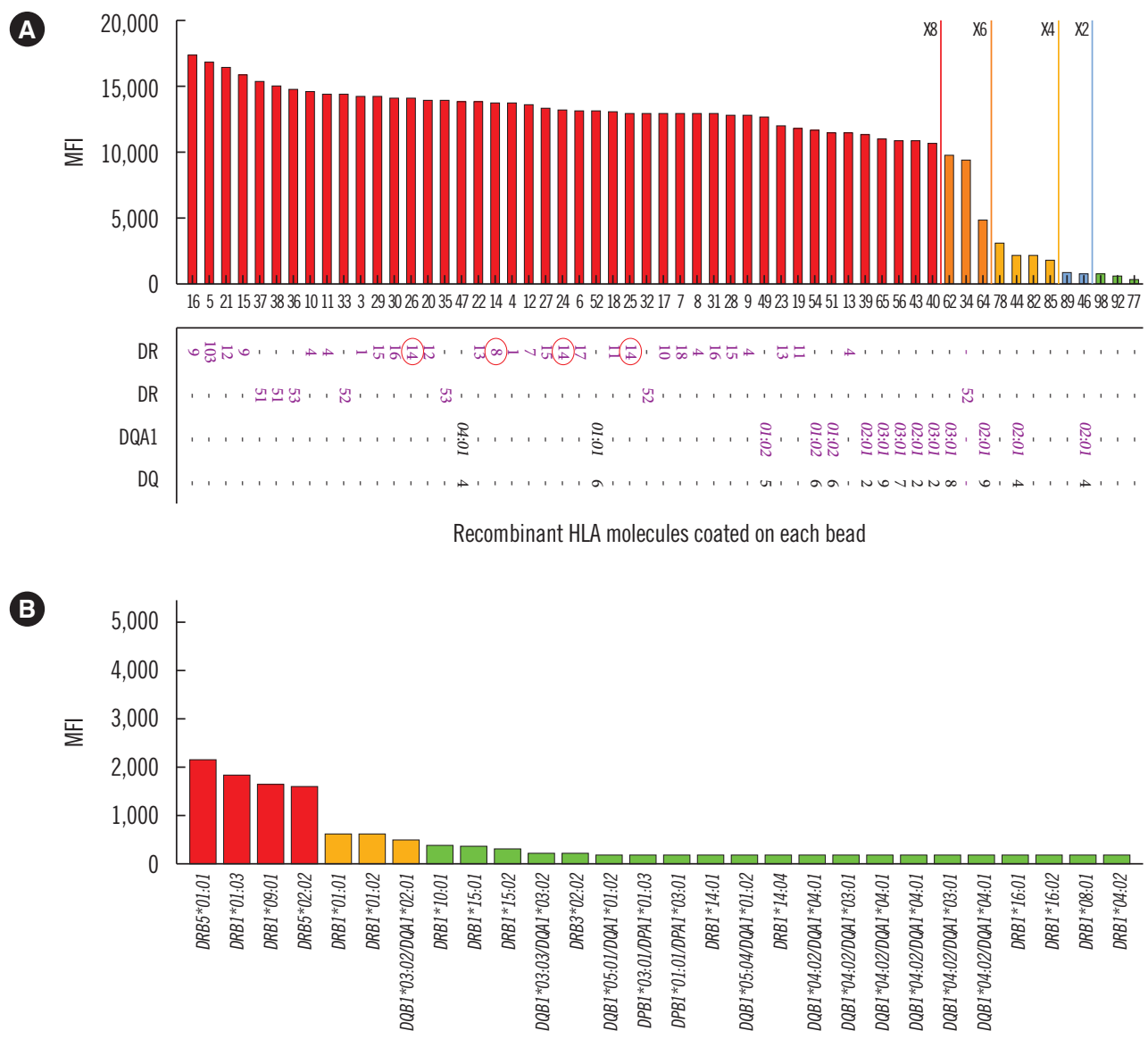

Recombinant HLA molecules coated on each bead

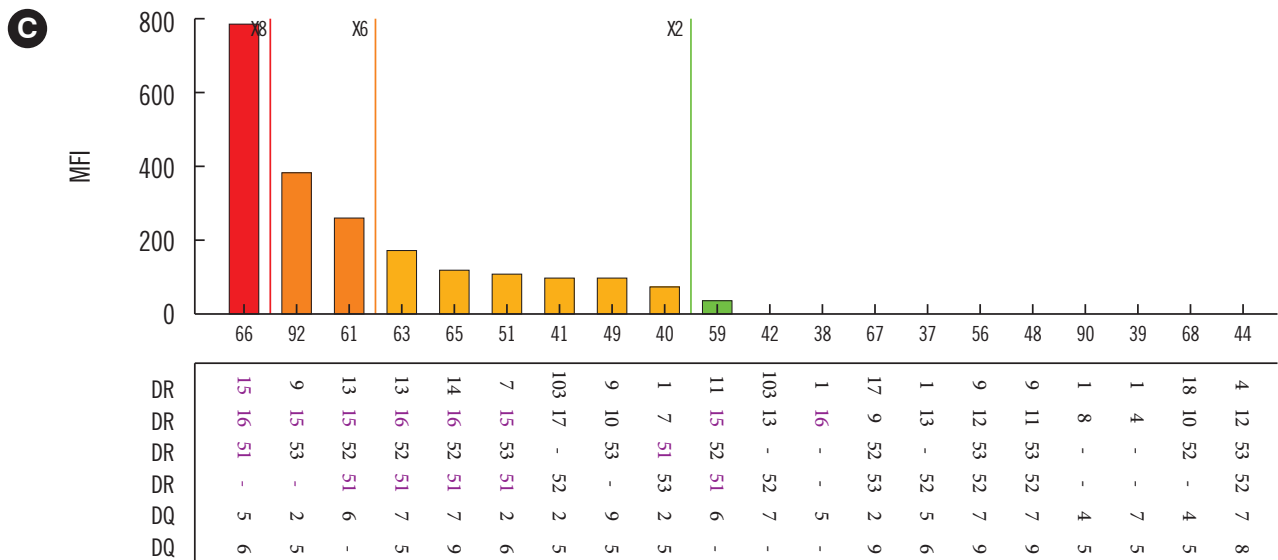

HLA antigens coated on each bead

Fig. 1. Reactivity patterns of class II HLA antibody assays. (A) LABScreen SAB class II (One Lambda, Canoga Park, LA, USA) SABA showing pan-HLA-DR positivity. Self-HLA-DR antigens are indicated by red circles. (B) Lifecodes class II SABA (Immucor, Stamford, CT, USA) showing positivity for HLA-DR51 and -DR9. (C) PRA Identification Bead Reaction (LABScreen PRA, One Lambda) showing weak positivity for HLA-DR51, DR15, and DR16.

Abbreviations: see Table 1.

HLA antibody screening (LABScreen Mixed, One Lambda, Canoga Park, CA, USA) was negative for class II HLA antibodies.
However, SABA using LABScreen SAB class II kit (One Lambda) showed strong pan-positive reactions [mean fluorescence inten- 
Table 1. Assignment of class II HLA antibody specificity according to bead type and manufacturer

\begin{tabular}{llc}
\hline Kit used & \multicolumn{1}{c}{ Class II HLA antibody specificity identified in each essay* } & Source of antigen \\
\hline One Lambda SABA (LABScreen SAB) & Strong: DR1, DR4, DR7, DR8, DR9, DR10, DR11, DR12, DR13, DR14, DR15, DR16, & Recombinant HLA molecule \\
& DR17, DR18, DR51, DR52, DR52, DR103, DQ2, DQ4, DQ5, DQ6, DQA1*01:01, & \\
& DQA1*01:02, DQA1*03:01, DQA1*04:01 & \\
& Moderate: DQ8, DQ9 & Recombinant HLA molecule \\
Immucor SABA (Lifecodes LSA) & Weak: DR51, DR103, DR9 & Purified human HLA \\
One Lambda screening (LABscreen Mixed) & Negative for class II HLA antibodies & Purified human HLA \\
One Lambda identification (LABscreen PRA) & Weak: DR15, DR16, DR51 & \\
\hline
\end{tabular}

*Strong, >10,000 MFI; Moderate, 3,000-9,999 MFI; Weak, 1,000-2,999 MFI.

Abbreviations: HLA, human leukocyte antigen; PRA, panel reactive antibody; MFI, mean fluorescence intensity; SABA, single antigen bead assay.

sity (MFI) $>10,000$ ] against all HLA-DR, including self-HLA antigens (Fig. 1A). The SABA results were not changed in the presence of the adsorption beads provided by manufacturers. The patient's serum was subsequently retested using a different SABA kit (Lifecodes LSA Class II Single Antigen Kit, Immucor), and this test revealed weak positive reactions (MFI 1,000-2,000) against DR9 and DR51 (Fig. 1B). The LABScreen panel reactive antibody (PRA) (One Lambda) assay, in which the beads are coated with purified human HLA, showed positive reactions against DR15, DR16, and DR51 (Fig. 1C). All assays were performed using the same initial serum, and the results were adjusted according to the results of the simultaneously tested negative control serum provided along with the kit.

Serologic evidence for the presence of donor-specific antibodies is an important criterion for Banff classification [7]. However, the presence of antibodies against denatured HLA has no clinical impact [8]. A previous study found antibodies against denatured class I HLA in 39\% of patients on the kidney transplant waiting list [3]. These antibodies were not considered to be targeting intact cells, but rather targeting the heavy chains of HLA without $\beta 2$-microglobulin, peptide, or cryptic antigens, as well as the polystyrene microbead itself $[2,4,9]$. Such unexpected antigenic targets may become exposed by conformational changes of HLA molecules induced by purification and bead coating of single-clone allelic HLA [2, 9], eventually resulting in false positive antibody reactions. Antibodies against denatured class II HLA have been associated with the female gender and systemic lupus erythematous [6]; by contrast, our patient was diagnosed as having membranous nephropathy.

In the present case, different HLA antibody reactions were observed with two SABA kits from different manufacturers (LABScreen, One Lambda and Lifecodes, Immucor). Antigenic coating process was not clearly described on the manufacturer's insert information. Immucor SABA did not show pan-DR reactivity with the sample that showed false positive reaction in One Lambda SABA (Table 1).

In this study, the screening (LABScreen Mixed) showed no antibody reactions and identification beads (LABScreen PRA) demonstrated weak positive reactions with HLA-DR15, -DR16, and -DR51 (Table 1). Both beads were coated with purified human HLA. However, the negative screening results may be due to the low sensitivity of the assay because of multiple class II HLA antigen beads being shared on a total of five beads. In addition, the sensitivity of an identification assay including class II HLA antigens on each bead could be lower than that of SABAs. For this reason, false positive reactions due to weak antibodies could not be demonstrated using screening or identification beads alone.

Flow-cytometric cross-matching to lymphocytes with known HLA antigens is another strategy to overcome false positive reactions. However, we could not perform any cross-matching tests, which is the major limitation of this study. Despite this limitation, our findings show that false positive reactions with antibodies against denatured class II HLA might differ according to the different SABA kits. This suggests an alternative approach to overcome false positivity in SABA caused by antibodies against denatured class II HLA.

\section{ACKNOWLEDGEMENTS}

None.

\section{AUTHOR CONTRIBUTIONS}

BGP, YP, and HSK conceptualized and designed the study. BGP and YP searched the scientific literature, analyzed the data, and 
Park BG, et al.

False reaction with denatured HLA antibody

wrote and revised the report. YSK, BSK, and HSK collected the data, supervised the study, gave administrative support, and revised the report.

\section{CONFLICTS OF INTEREST}

None declared.

\section{RESEARCH FUNDING}

None.

\section{ORCID}

Borae G. Park

https://orcid.org/0000-0001-9710-9253

Younhee Park https://orcid.org/0000-0001-8458-1495

Beom Seok Kim https://orcid.org/0000-0002-5732-2583

Yu-Seun Kim https://orcid.org/0000-0002-5105-1567

Hyon-Suk Kim https://orcid.org/0000-0001-5662-7740

\section{REFERENCES}

1. Lobashevsky AL. Methodological aspects of anti-human leukocyte antigen antibody analysis in solid organ transplantation. World J Transplant 2014;4:153-67.

2. El-Awar N, Terasaki PI, Nguyen A, Sasaki N, Morales-Buenrostro LE, Saji $\mathrm{H}$, et al. Epitopes of human leukocyte antigen class I antibodies found in sera of normal healthy males and cord blood. Hum Immunol 2009;70:844-53.

3. Visentin J, Guidicelli G, Bachelet T, Jacquelinet C, Audry B, Nong T, et al. Denatured class I human leukocyte antigen antibodies in sensitized kidney recipients: prevalence, relevance, and impact on organ allocation. Transplantation 2014;98:738-44.

4. Morales-Buenrostro LE, Terasaki PI, Marino-Vazquez LA, Lee JH, ElAwar N, Alberu J. "Natural" human leukocyte antigen antibodies found in nonalloimmunized healthy males. Transplantation 2008;86:1111-5.

5. In JW, Rho EY, Shin S, Park KU, Song EY. False-positive reactions against HLA class II molecules detected in Luminex single-antigen bead assays. Ann Lab Med 2014;34:408-10.

6. Grenzi PC, de Marco R, Silva RZR, Campos EF, Gerbase-DeLima M. Antibodies against denatured HLA class II molecules detected in Luminex-single antigen assay. Hum Immunol 2013;74:1300-3.

7. Roufosse C, Simmonds N, Groningen MC, Haas M, Henriksen KJ, Horsfield C, et al. A 2018 reference guide to the Banff classification of renal allograft pathology. Transplantation 2018;102:1795-814.

8. Visentin J, Marroc M, Guidicelli G, Bachelet T, Nong T, Moreau JF, et al. Clinical impact of preformed donor-specific denatured class I HLA antibodies after kidney transplantation. Clin Transplant 2015;29:393-402.

9. El-Awar N, Lee JH, Tarsitani C, Terasaki PI. HLA class I epitopes: recognition of binding sites by mAbs or eluted alloantibody confirmed with single recombinant antigens. Hum Immunol 2007;68:170-80. 